\title{
SOFT COMPUTING TECHNIQUE FOR PRODUCT DESIGN SUGGESTION IN SMART MANUFACTURING INDUSTRY
}

\begin{tabular}{|l|l|}
\hline Jitesh Kumar Khatri & Jyoti Kumar \\
Indian Institute of Technology, Delhi & Indian Institute of Technology, Delhi \\
Delhi, India & Delhi, India \\
jiteshkkhatri@gmail.com & meetjyoti@gmail.com \\
\hline
\end{tabular}

\section{Abstract}

With the phenomenal growth in consumer durables industry, and more than ever before change and dynamism in the variety of design needs within the constraints of very limited time and investment, the urgent need of design automation is being recognized all over the world. To be able to capture the market through proactive launching of customer desirable products, the whole development cycle needs to be shrunk without any compromise of product features. This work suggests a fuzzy logic and ANN based noble design suggestion system which would assist the designers with a range of $3 D$ design suggestions to choose from and take forward based on variable inputs from the market. This would facilitate faster designs through automation, reducing the overall design cycle time. The inputs for the design suggestions are based on anthropometry study and emotional behavior. Fuzzy logic is an efficient tool to choose the impact factor of a suggestion from a particular methodology based on the associated membership function and ANN has good learning capability and combination of both forms an efficient system for design suggestion.

\section{Keywords-Design Automation, Development cycle time.}

\section{INTRODUCTION}

Over the past few decades, there has been a tremendous increase in the production capabilities of manufacturing industries through the process of automation [1]. This automation and productivity enhancement can be easily seen in almost all the sectors except a few like construction industry [2] \& design where the automation practices have not so vastly impacted the production efficiencies. There has been for example, monumental automaton in shoe industry over the past few decades [3]. The capability of manufacturing industries have increased however, there are also more than ever frequent changes in product designs in the market. Newer and customer friendly products are flooding the market and the number of competitors has also increased considerably. To secure market share, the manufacturer would like to launch his product ahead of its competitors. The advances in almost all the technologies all over has made products very complex than they were ever before, which requires specialist expertise and potentially time-consuming product development cycles.

The profit margins are narrowing in almost all sectors and will continue to become even lesser. Even the products where margins are better, cost cutting is mandatory due to competition and global outsourcing [4]. A lot of effort has been done for cost cutting through process time optimization or cutting the cost of engineering activities. Almost all of these methods are specific solutions which might be locally powerful and helpful for specific products and processes. Design automation, could serve as a very powerful and efficient tool to dramatically cut costs. Although electronic design automation is now very user friendly [5] and result oriented, only a very little progress has been done in the area of form design. The field of form design automation is very old, as old as 1980s, but most of industries even now depend largely upon manual designs only.

After interviewing some of the members of industrial design teams at a few companies, it was understood that most designers in area of form design still feel that form design automation might not be a possible \& practical solution capable of solving their problems of long lead time that goes in manual designs. Some designers however showed their keen interest and helped to explore the segments of the form design process that can be automated and thus save lots and lots of time. The proposed automation in form design could cater to the worlds faster changing design requirements and thus reduce pressure on the industrial designers for faster and frugal designs.

The idea of form design automation was very well received by the capitalists who showed the hopes of capturing the market by becoming the early product introducers to the market and 


\section{(2) \\ ELK \\ Asia Pacific Journals}

also as a cost innovation tool which is very much indispensible to grow and even to sustain in today's scenario of tough competition with so many market players coming in.

\section{AN INTELLIGENT AUTOMATION APPROACH FOR DESIGN AUTOMATION}

The proposed form suggestion system proposes to convert the information obtained from the customers in raw form and the result is generation of $183 \mathrm{D}$ forms which the designer can choose from and take towards finalizing the design aligned as per user stated and unstated needs. This is accomplished though use of fuzzy FIS editor and ANN as classification platform and CATIA as the design platform.

\section{A. Understanding the user needs}

The first step in providing the user appealing product form is to understand the requirements of the user. In many cases the information available for product design would not be sufficient to decide upon all the details of the design and one has to use various studies and analysis to come to the conclusion. There are many ways of understanding the customer needs including the questionnaire, market study, observation and home visits etc. It is clear that the inputs and likings from these sources would be highly variegated and would need systematic analysis. In our research work we confined the design automation work to the automatic design of water bottle based on some rough inputs from the customer.

\section{B. Anthropometric Study}

Anthropometric measurements are a great help to understand the optimal dimensions of a product according to the user group for the best and most comfortable user experience. The sizes or dimensions of hand are important in any consideration of hand function [6]. Handles of tools or objects to be handled by hand must be suitably sized. Information on the range of variation in the sizes and dimensions comprises a very important knowledge of human body represented by anthropometric data. Our subject of designing of a user appealing bottle design has a very important requirement of knowing the most suitable dimensions like the bottle length and diameters and consequently the volume also.

In our work, we have classified the hand anthropometric data as per the user age and sex. This gives us a good idea of what the most suitable object dimensions would be.

\section{$C$. Uncertainty in parameter suggestion.}

There are many other factors which very significantly contribute to the design making process which includes not only the dimensions but also factors like colour, texture, looks etc. These factors also vary as per the age group, sex and other usage consideration. Different methodologies have been used to understand the suggestions from these contributing factors and it is observed that each dimension/ colour suggestion could be differently suggested by the various related methodologies. Colour for example may be suggested based
ELK Asia Pacific Journals - Special Issue ISBN: 978-81-930411-4-7

on age and also on basis of sex. We need a classification or algorithm as a decision making process in this complexity.

\section{The fuzzy approach}

It's said that a thorn removes a thorn. Fuzzy Logic is a simple robust technique developed to allow formal management of uncertainty and imprecision [7]. It allows effective decision making in the presence of uncertainty.

As there are so many factors affecting the dimensions and the colour of the product simultaneously, it is important to chose which factor should be the predominating factor affecting the decision and to what degree. The nature of this dependence is non linear and the different fuzzy membership functions are very suitable to deal with the uncertainty.

\section{E. CATIA as the design platform}

CATIA offers a solution to model complex and intelligent products through the systems engineering approach [8]. It covers the requirements definition, the systems architecture, the behavior modeling and the virtual product. CATIA in the present scope of work has good capability of mapping data in excel format to 3D point locations on the screen. These points facilitate the dimensions of the various proposed output forms. The designs forms thus generated in CATIA are suitable for modelling and finally to be fed to the manufacturing unit. CATIA also facilitates engineering evaluation of designed products which is a later scope of extension of present work.

\section{AN ILLUSTRATION OF PROPOSED APPROACH}

The work for our chosen design automation for a user appealing water bottle began with understanding of the user needs. This was one of the most important considerations as this would guide the whole decision making process. The inputs from the market are generally not clear and we had to classify into our broader classification based on age, sex and purpose. We have divided our object into a series of heights $(\mathrm{H} 1, \mathrm{H} 2, \mathrm{H} 3 \ldots . \mathrm{H} 7)$ and (D1, D2, D3....D8) diameters. The proposed output would be a series of designer usable 3D forms which the designer can take forward and further refine to the final design.



Fig. 1. Bottle classified with series of heights \& diameters

The said series of designer usable 3D form designs in CATIA would be the outputs which would be most suitable for the users and have been decided with the help of Fuzzy Inference System based on the inputs which are just a set of age, sex and 


\section{(2) \\ ELK \\ Asia Pacific Journals}

usage type of the user. For example, the problem statement could be "to design a picnic water bottle for a girl of class 3 ". This simple statement has got all the information that we need in this study to suggest around 18 3D bottle designs suitable as per user needs. One or more of these 18 designs could be selected by the designer (or the user in case of user availability) and taken forward to be refined to the final design. Fig 2 shows the proposed automation system blocks.

To understand the requirements at each stage to get the desired 18 design forms, a reverse engineering approach is helpful. Any 3D form in CATIA would be a number of points with their space coordinates and also some data representing detail of colour etc. To be able to obtain the said 18 design forms in CATIA, we would be in need of all these points and colours per design of bottle. If we are able to have these points and colour details we could use algorithms to join them to have the desired form. Now how to get these points fixed. As per age, sex and purpose of use, there would be changes in the size (Height and Diameters) and also change in colour preferences.

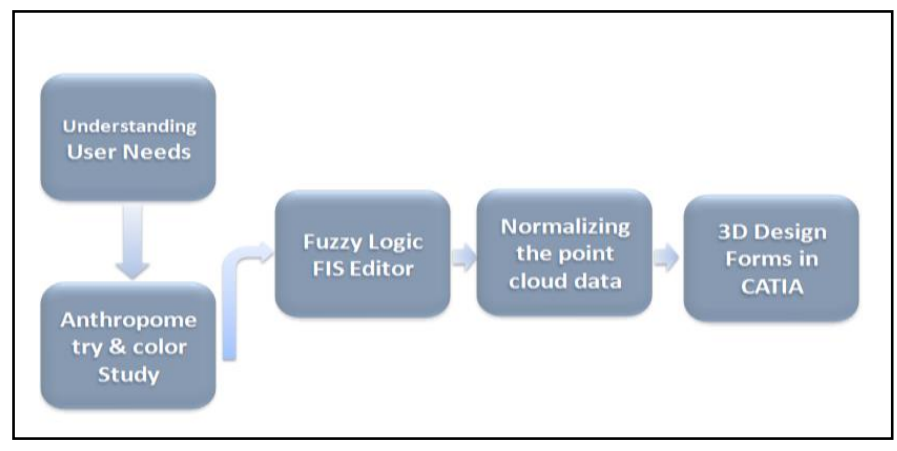

Fig. 2. The proposed design automation process

The major details on dimensions front are achieved through data resources on anthropometry and survey on Anthropometric dimensions of hand for ages from 1 to 50 years. As the hand and arm dimensions are different as per age so the suggested height of bottle would also change as per age.

Similarly the diameters would also vary as per age Fig 3 below shows the various dimensions measured in hand and how hand dimensions would affect the dimensions of objects for a comfortable handling posture.

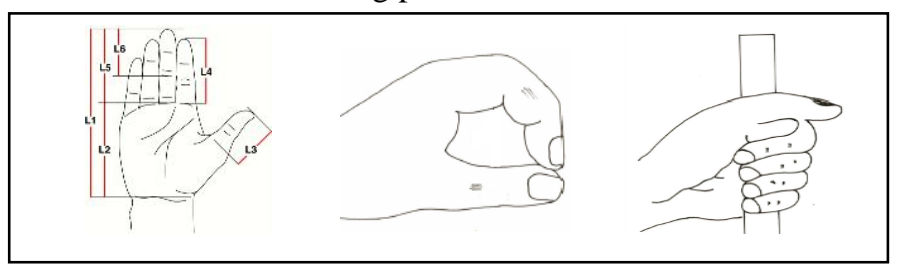

Fig. 3. Anthropometric studies of hand dimensions.

The anthropometric dimensions of males however would generally be different than females for the same age also. Females would normally have less broad hand dimensions than males.

\section{ELK Asia Pacific Journals - Special Issue}

ISBN: 978-81-930411-4-7

Colour preference similarly has a dependence on age and also sex. There is a cycle of changing colours that affects our mind and body through the different stages of life. These are reflected in our changing colour preferences. Children have colour likes and dislikes according to individual character and stage of development [9].

Over a hundred years ago the pioneering educationalist Rudolph Steiner believed that people were surrounded by particular colours that had a spiritual influence and objective effect on their emotional life as well as benefiting physical health and mental well-being.

Strong, bright colors have the effect of shocking the baby's inner vibrations, which can make the baby unsettled and restless. Comfort colours for very young children are pinks, peaches, pale almond beiges and lavenders as these equate to love and security [9]

Teenagers however like to go more for black and black like shades. They are generally affected by identity crisis.

Becoming old can bring about a sense of loneliness and fear so decorating with the elderly in mind needs to address warmth, security and harmony. Variety in the colours in the immediate environment can boost interest in the world and keep cognitive function alive. Older people can be drawn to soft pastels but they may not have the vitality of hue needed to stimulate the mind and mood. Eyesight problems can also impair how the colour is seen and what is seen [9]

Softer shades of reds and oranges are warming and can help with circulation and energy levels. Peaches, apricots, warm tans, and pinks can also be used for this purpose.

The problem faced in the decision making process is that in this case, where the different factors are impacting the decision of a particular output. Color or dimensions, which factor will impact the decision \& to what degree Taking one example to understand this complexity. Color suggestion as discussed above varies with age.

Also it varies with sex. Now for very smaller age group different colors (with in favorable category for that age) would not be impacted by sex as children irrespective of male/ female would have a similar color preference. However, for teenagers, the impact of sex on the color decision would be tremendous. So the membership function of color with respect to age would be like the one shown in Fig 4 below (the Gaussian MF)

Fuzzy logic helps to resolve this state of uncertainty. Which factor in decision making with influence the output to what extent would be based upon that input and the membership function of that input in the decision making process. For example color decision with age has a Gaussian type membership function. Similarly, we have defined membership functions for other influencing as well 


\section{ELK}

\section{Asia Pacific Journals}


Fig. 4. Fuzzy Membership functions enabling to deal with the uncertainity.

The output of the decision of influencing factors for color \& dimensions through FIS is converted into point cloud data. The outputs of FIS are then mapped to final values through rationalization process.

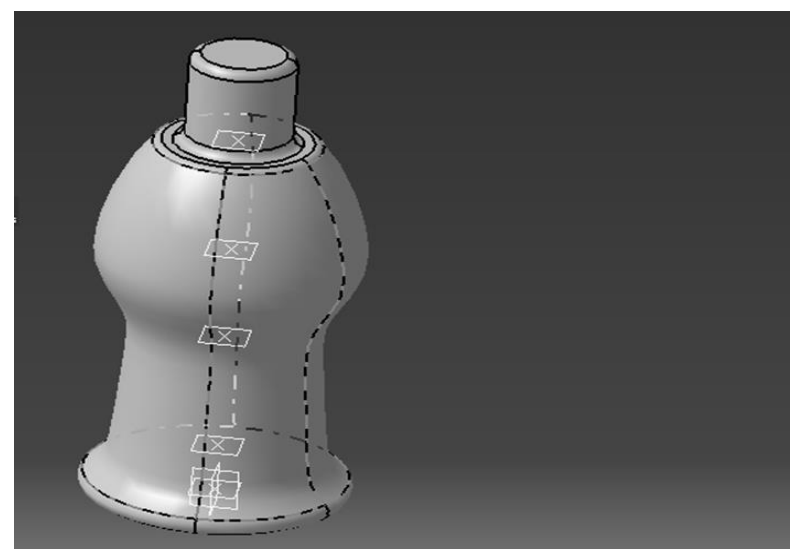

Fig. 5. Anthropometric studies of hand dimensions.

The finalized point cloud data serves as the required coordinates of points in $3 \mathrm{D}$ which would be able to produce 3D forms on CATIA screen. Since in place of specific points as suggestion, we would generally be suggested with ranges, the algorithm taking different permutations of points within this range would be producing the required 18 3D forms as desired. The designer can now choose any one or more of these design forms and adjust it to make the final desired output form.

\section{FINAL REMARKS}

Design suggestion through the said process of automation can significantly assist the designer and can save lot of stress on designer in addition to design cycle time reduction and thus capturing the market through early introduction. Preliminary study of process validation has been done with the help of bottle design and we hope to refine it through further work and bring it to a practicable level which the designers and capitalists would adore and would be largely benefited. Fuzzy logic based FIS with its powerful discriminating capability in uncertainty supports the decision making process for suggestion of dimensions and colours. CATIA has variegated capabilities which make it a suitable platform for design automation. Along with adorable foam design capabilities it also has its strength in evaluating engineering parameters which could be instrumental in further usability of this work.
ELK Asia Pacific Journals - Special Issue ISBN: 978-81-930411-4-7

\section{Acknowledgment}

The authors would like to gratefully acknowledge the kind support of Mr Kamal Panduit who helped us to arrange extensive interviews with the designers.

\section{References}

[1] IEEE ICIT '02. 2002 IEEE International Conference on Industrial Technology. 'Productivity Reincarnation through Robotics and Automation'(Cat. No.02TH8654)

[2] Matt M Hewitt, John A Gambatese, "Autoation Consideration during project design"

[3] Monckton, S.P., Chrystall, K.” Design and development of an automated footwear testing system, Robotics and Automation, 2002. Proceedings. ICRA '02. IEEE International Conference.

[4] Ian DOMOWITZ, "Industry Margins \& Business Cycle", Economic Letters 19(1985) 73-77

[5] Robert Brayton, Jason Cong, "Electronic Design Automation Past, Present, and Future" NSF Workshop, July 8-9, 2009

[6] Arunesh Chandra, Pankaj Chandna \& Surinder Deswal, "Analysis of Hand Anthropometric Dimensions of Male Industrial Workers of Haryana State" International Journal of Engineering (IJE), Volume (5) : Issue (3) : 2011

[7] T.Ross, Fuzzy Logic with Engineering Applications, Mc Graw- HillInc; 1995.

[8] Nikhil R. Chaple1, Dr. T.R. Deshmukh, "Design and Optimization of piston using finite element analysis" International Journal of Research in Science \& Technology (IJRST), Volume 1, Issue 2, April 2014:Page No. $4-6$.

[9] http://www.resene.co.nz/homeown/use_colr/colours-for-living.htm

[10] http://www.business-standard.com/article/news-cm/southern-stateshigh-in-rural-consumer-durables-consumption-assocham-study 